Cinémas

Revue d'études cinématographiques

Journal of Film Studies

\title{
JOST, François. Un monde à notre image. Énonciation, cinéma, télévision. Paris : Méridiens Klincksieck, 1992, 140 p.
}

\section{Serge Cardinal}

Volume 5, numéro 3, printemps 1995

URI : https://id.erudit.org/iderudit/1001154ar

DOI : https://doi.org/10.7202/1001154ar

Aller au sommaire du numéro

Éditeur(s)

Cinémas

ISSN

1181-6945 (imprimé)

1705-6500 (numérique)

Découvrir la revue

Citer ce compte rendu

Cardinal, S. (1995). Compte rendu de [JOST, François. Un monde à notre image.

Énonciation, cinéma, télévision. Paris : Méridiens Klincksieck, 1992, 140 p.]

Cinémas, 5(3), 181-185. https://doi.org/10.7202/1001154ar d'utilisation que vous pouvez consulter en ligne.

https://apropos.erudit.org/fr/usagers/politique-dutilisation/ 
JOST, François. Un monde à notre image. Énonciation, cinéma, télévision. Paris: Méridiens Klincksieck, 1992, 140 p.

Dans la foulée des recherches cinématographiques récentes, maintenant soucieuses de tracer la géographie des trajets, parcours et positions de compréhension du spectateur, François Jost, dans son dernier ouvrage, soumet les questions entourant l'énonciation au cinéma à la lumière et à la rumeur des faits de réception. En bref, il resitue "[...] l'énonciation audiovisuelle dans un cadre communicationnel" (p. 12).

Cette résonance avec ce large courant - qui va de la sémiopragmatique aux sciences cognitives - n'est pas sans produire une dissonance avec les théories consacrées de l'énonciation. Plus précisément, les insuffisances de ces théories à expliquer les "illusions " nécessaires à la compréhension du récit par le spectateur invitent François Jost à revoir un refoulé, l'anthropomorphisme, et à réhabiliter une figure longtemps muette, l'auteur.

Cette réévaluation prend d'abord la forme d'une mise à distance. D'abord, de la thèse défendue par André Gaudreault ' qui, tout en cherchant à éviter l'anthropomorphisation, développe un tableau des instances logiques responsables du récit arrimé aux phénomènes de communication. Or, selon Jost, cette thèse souffre d'une ambiguïté insoluble dans la mesure où "[...] l'idée même de communication requiert que l'on sorte de la pure immanence textuelle [...]" (p. 20). Sinon, l'on se condamne à considérer l'activité de spectature uniquement comme une simple activité de décodage, tout en restant aveugle aux jeux de la focalisation, aux " [...] variations de la régulation des informations narratives [...]" (p. 20) dont tout spectateur a l'intuition.

Un monde à notre image tente aussi de se situer face à la théorie de l'énonciation chez $\mathrm{Metz}^{2}$, qui poussait l'autonomie du texte 
jusqu’à aboutir "[...] à une énonciation non "attribuée" [...]" qui " [...] passe par l'idée d'un machinisme textuel, autonome, stable $[\ldots]$ ", indépendant des constructions imaginaires des spectateurs. Cette position qui rejette chaque lecture dans un écart variable par rapport à l'immanence textuelle en forme de vérité, s'oppose fondamentalement au projet de Jost, cherchant justement à prendre en compte la mouvance de la spectature.

Une narratologie visant à définir les principes constitutifs du récit cinématographique ou la solitude d'un machinisme textuel ne peut relever adéquatement les phénomènes de compréhension d'un acte discursif (le film) inscrit dans un schéma communicationnel ; elle ne peut rendre compte à la fois de la diversité et de la mobilité des lectures du film liées à l'attribution d'une intentionnalité à la dérive des horizons d'attente spectatoriels :

Les images cinématographiques nous disent quelque chose, non parce qu'elle "parlent ", mais seulement parce que, au travers de "ces traces qui nous ressemblent ", comme dirait Godard, nous percevons une visée communicative [...]

[Les images] sont fabriquées dans l'intention de nous dire quelque chose $[. .$.$] le film est un acte de langage$ que le spectateur doit interpréter (p. 11-12).

Comme la nature chez $\mathrm{Claudel}^{3}$, le film prend sens dans la mesure où une force organisatrice est reconnue dans la donation des images, dans la mesure où le spectateur inscrit une intentionnalité dans une suite d'images et de sons. La reconnaissance de cette intentionnalité est favorisée par l'encadrement institutionnel du film et se trouve cristallisée dans la figure de l'auteur. Mais surtout, la reconnaissance de cette intention semble d'abord concerner le statut artistique du film avant de faciliter la compréhension du récit. Avant de régler les difficultés de structuration narrative, l'intentionnalité décide du mode de saisie d'un complexe audiovisuel: est-ce une œuvre filmique ou un simple enregistrement? Ce qui chez Jost, prolongeant en cela Arthur Danto ${ }^{4}$, signifie : ces images appellent-elles la production de sens ou ne sont-elles que vulgaires reproductions machiniques? 
Dans ce cadre communicationnel balisé par des courants de lecture anthropomorphisants, la figure de l'auteur occupe une place centrale. Bien sûr, l'auteur qui intéresse François Jost n'est plus ce centre romantique sanctionnant en définitive les interprétations périphériques gravitant autour de son double: le film. Ici, l'auteur est une construction hypothétique transformant une machine de vision en une œuvre générant une multitude " [...] d'intentions humaines inférées [...]" (p. 71). L'auteur, chargé de "[...] significations construites à partir du paratexte comme des épitextes divers [...]", devient un outil de compréhension du récit, comme le sont, selon une logique différente, les notions de genre ou de figures rhétoriques.

Dans la perspective d'un spectateur au poste de commande, il devient possible à la narratologie de prendre acte des variations cognitives dont l'imputation au seul "méganarrateur" chez Gaudreault, par exemple, ne permettait pas de saisir. Il est alors permis de faire la différence entre le partage réglé d'un même "environnement cognitif pragmatique" entre le spectateur et un personnage (dans le flash-back entre autres) et une paralepse (une infraction à la focalisation adoptée en cours de récit et octroyant un surplus de savoir au spectateur). La paralepse, notamment, met en évidence la mobilité de la spectature entre le texte, le narrateur implicite et l'auteur. Devant cette infraction, le spectateur fait le choix ou de considérer la paralepse comme pertinente et alors il induit " [...] une intention informative de celui qui raconte" (p. 46) ou au contraire "[...] la pertinence artistique guide la réception du film et l'on considère que ces infractions sont des erreurs indignes d'un auteur conscient du matériau qu'il travaille" (p. 57). Dans un cas comme dans l'autre, l'intentionnalité inférée devient un outil de compréhension et de jugement.

L'acte de langage que constitue le film est donc déterminé en trois étapes: 1) l'auteurisation du document audiovisuel menant à la reconnaissance du document comme émis par des "êtres qui me ressemblent" ; 2) l'identification de l'auteur et 3) la reconnaissance de l'intention qui influencent " [...] l'hypothèse que le spectateur formule lorsqu'il y a contradiction narrative [...]" (p. 66-69).

Un monde à notre image. Énonciation, cinéma, télévision 
La complexité de la spectature ne s'arrête pas là. Dans le passage de la vue à la prise de vue jusqu'au regard, du représenté à l'acte d'enregistrement jusqu'à la vision d'un personnage, le film multiplie des "indices ostensifs" qui renvoient aux multiples masques de l'énonciation. "Force est de constater, une fois encore, que le film ne construit pas un schéma de communication simple dans lequel un destinateur unique s'adresserait à un destinataire" (p. 77). L'interprétation d'une suite d'images se trouve modifiée par les masques que le spectateur décerne à une énonciation polyphonique. Ainsi, chaque acte d'ostension n'ayant pas de pertinence au niveau diégétique renvoie au supposé-réalisateur, alors qu'un point de vue partagé par le spectateur excédant l'environnement cognitif des personnages renvoie au narrateur-implicite. Mais lorsque le tremblé, le flou ou un angle marqué affectant la prise de vue ne "[...] sont renvoyés ni à des intentions discursives (= supposé-narrateur) ni à des intentions narratives ( $=$ narrateur), mais au contexte d'énonciation [...]" (p. 82), le spectateur rejoint le filmeur empirique. Finalement, lorsqu'il s'agit de reconnaître une intention de communiquer, nous retrouvons l'auteur construit. Cette dérive inférentielle de la part du spectateur, que retrace François Jost, souligne l'influence d'un anthropomorphisme à plusieurs vitesses dans la mise en forme du récit et sa réception.

Mais si le spectateur peut être un sujet narrativisant, "[...] il peut aussi aller de l'énonciation narrative à l'énonciation cinématographique ou filmique dans un aller retour [...] C'est dans ce mouvement que l'on trouvera le film comme ouvre" (p. 119). Le spectateur quittant la nécessité narrative diversifie son expérience du film, à condition, bien sûr, qu'il construise "une intentionnalité artistique" (p 124). Ici, l'entreprise narratologique de François Jost n'est plus simplement concernée par des parcours unanimement dirigés vers une compréhension du récit, elle n'est plus simplement attachée à dresser des bornes et des positions, elle est intéressée aux forces qui les dépassent et les déplacent. Reprochant à la théorie du cinéma de s'être limitée à l'étude de la fiction dans l'unique objectif de saisir de quelle manière elle était comprise par le spectateur, le dernier chapitre d'Un monde à notre image ouvre sur les rapports entre l'inten- 
tionnalité et le statut artistique du film. Encore une fois, ce ne sera pas le texte filmique dans son autonomie réclamée qui décidera de ce statut ni même une reconnaissance à sens unique de l'auteur comme artiste :

Pour que le film soit une œuvre, il ne suffit pas que l'auteur ait organisé les différentes strates énonciatives [...] autour d'une intention esthétique, il faut encore que le spectateur, quittant la seule nécessité narrative lui permettant de comprendre le récit, cherche une autre logique (p. 131).

Il semble bien que cette autre logique soit aussi le point de fuite attirant à lui la recherche de François Jost qui, après la description des chemins, cherche à saisir les forces qui les traversent.

Serge Cardinal

Université de Montréal

\section{NOTES}

1 André Gaudreault, Du littéraire au filmique. Système du récit (Paris: Méridiens Klincksieck, 1988).

2 Christian Metz, L'Énonciation impersonnelle ou le site du film (Paris: Méridiens Klincksieck, 1991).

3 " "Jadis au Japon comme je montais de Nikkô à Chuzenji, je vis, quoique grandement distants, juxtaposés par l'alignement de mon œil, la verdure d'un érable combler l'accord proposé par un pin " [...]. Sans aller plus loin que la perception: est-ce un texte, ce qui ne parle que quand l'œil a trouvé le "point de vue", quand mon regard est devenu le regard à qui les choses sont dues? [...]. Que le monde soit à lire signifie brutalement qu'un Autre, de l'autre côté, écrit les choses données, et qu'avec un bon angle de vue, en principe, je pourrais le décrypter. " Jean-François Lyotard, Discours, figure (Paris: Klincksieck, 1971, p. 9).

4 Arthur Danto, La Transfiguration du banal (Paris: Seuil, 1989). 